\title{
ANÁLISIS DE DESLIZAMIENTOS SUBACUÁTICOS EN DELTAS LACUSTRES (LAGO NAHUEL HUAPI, ARGENTINA) A PARTIR DE BATIMETRÍAS DE ALTA RESOLUCIÓN
}

\author{
DÉBORA BEIGT ${ }^{1 *}$, GUSTAVO VILLAROSA ${ }^{1,2}$, \\ EDUARDO A. GÓMEZ

\begin{abstract}
'Instituto de Investigaciones en Biodiversidad y Medioambiente (CONICET/UNComa),
S. C. de Bariloche, Río Negro, República Argentina.

${ }^{2}$ Universidad Nacional del Comahue, Centro Regional Universitario Bariloche, República Argentina.

${ }^{3}$ Instituto Argentino de Oceanografía (CONICET/UNS), Bahía Blanca,
\end{abstract} \\ Buenos Aires, República Argentina.
}

RESUMEN. Las localidades asentadas en las costas de los grandes lagos de Norpatagonia Andina están expuestas a frecuente actividad sísmica y volcánica regional y a fenómenos tsunamigénicos asociados. Olas extraordinarias como el tsunami de 1960 en el lago Nahuel Huapi, que afectó las costas de la ciudad de Bariloche, pueden ser inducidas por deslizamientos subaéreos o subacuáticos desencadenados durante un sismo de magnitud (Villarosa et al., 2009). Este trabajo aborda el estudio de los procesos de remoción en masa que se desarrollan en la porción sumergida de deltas lacustres ubicados en las costas de las localidades de Bariloche y Dina Huapi, con el fin de aportar información relevante que sirva de base a futuras evaluaciones de peligrosidad en estas costas. Se registró la morfología subacuática mediante un Sonar Batimétrico por Medición de Fase y se confeccionaron Modelos Digitales de Elevación y mapas derivados utilizando ArcGIS. Los resultados indican que se trata de deltas tipo Gilbert: frentes deltaicos de pendientes pronunciadas donde el sustrato se moviliza mediante procesos gravitacionales (deslizamientos, flujos de detritos y/o corrientes de turbidez). La abundancia y magnitud de los deslizamientos (con escarpas de cabecera de unos $10 \mathrm{~m}$ de altura y hasta $200 \mathrm{~m}$ de longitud y escarpas laterales que se extienden hasta el límite del área estudiada, superando los $600 \mathrm{~m}$ de longitud) indican que periódicamente se desarrollan fenómenos de remoción que involucran grandes volúmenes de sedimento. Estos eventos son potenciales generadores de ondas en la superficie del lago. La cercanía entre estos ambientes y las localidades costeras implica que un evento de este tipo desencadenado por un terremoto de magnitud considerable en la región podría afectar a las costas más vulnerables. 
Subaequeous landslides analysis in lacustrine deltas (Nahuel Huapi lake, Argetnina) through high-resolution bathymetry

\begin{abstract}
The cities located along the coasts of the North-Patagonian Andean lakes are exposed to regional seismic and volcanic activities and associated tsunamigenic events developed in the lacustrine basins. Extraordinary waves, such as the 1960 tsunami in Nahuel Huapi lake that hit the coasts of the city of Bariloche, may be induced by subaequeous or subaerial landslides triggered during a strong earthquake (Villarosa et al., 2009). This article focusses on the study of mass-wasting processes that occur in the submerged portion of lacustrine deltas located at the coasts of Bariloche and Dina Huapi cities. The results presented here are an input for future hazard evaluation studies. The subaequeous morphology was surveyed using a Phase Measuring Bathymetric Sonar System. Digital Elevation Models and DEMderived maps were performed in ArcGIS. Bathymetric data show characteristic features of Gilbert-type deltas: steep delta fronts where sediments are mobilized by gravitational processes (landslides, debris flows and/or turbidity currents). Numerous landslides found in the delta fronts ( 10-m high headscarps with a maximum length of $200 \mathrm{~m}$, and lateral scarps that exceed a length of $600 \mathrm{~m}$ ) indicate that mass-wasting phenomena involving large volumes of sediment periodically develop in these environments. These events are potential generators of waves in the water surface. The proximity among these deltas and the coastal cities implies that an event of this kind triggered by a strong regional earthquake could affect their vulnerable coasts.
\end{abstract}

Palabras clave: lago Nahuel Huapi, movimiento en masa, deslizamientos subacuáticos, deltas lacustres, tsunami 1960.

Key words: Nahuel Huapi lake, mass-wasting events, subaequeous landslides, lacustrine deltas, 1960 tsunami.

*Correspondencia: Instituto de Investigaciones en Biodiversidad y Medioambiente (CONICET/UNComa), S. C. de Bariloche, Río Negro, Argentina. E-mail: dbeigt@ comahue-conicet.gob.ar

\title{
1. Introducción
}

Los deslizamientos en cuerpos lacustres son reconocidos, junto a los flujos gravitatorios, como importantes medios de transferencia y depositación de sedimentos en estos ambientes (Baster et al., 2003). Estos eventos, ya sea que se desarrollen en un ambiente subaéreo adyacente a la cubeta lacustre o en el fondo del lago, impactan en el lecho modificando su topografía de forma brusca, siendo potenciales generadores de ondas tipo tsunami en la superficie de estos cuerpos de agua. 
Investigaciones efectuadas en los lagos Ginebra (Kremer et al., 2012) y Brienz (Girardclos et al., 2007), en Suiza, muestran la importancia del estudio de estos procesos particularmente en los ambientes deltaicos, donde las elevadas pendientes y las altas tasas de sedimentación favorecen la movilización del sustrato. En ambos trabajos se hallaron evidencias de depósitos de remoción en masa (turbiditas) en la porción más profunda de los lechos lacustres, generados a partir del colapso de deltas. El movimiento en masa ocurrido en 1996 en un delta del lago Brienz fue producto de la acumulación normal de sedimentos en este ambiente, mientras que en el lago Ginebra, el colapso se vinculó con un desprendimiento rocoso subaéreo producido en la cuenca del Ródano en el año 563, que afectó al delta. Ambos eventos provocaron ondas de tsunami, y en el lago Ginebra los pueblos ribereños y la antigua ciudad de Ginebra se vieron afectados por esta ola, que produjo un importante número de víctimas mortales.

\section{2 Área de estudio}

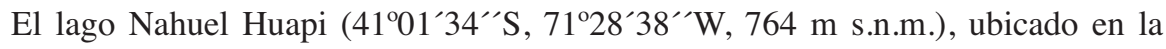
Norpatagonia andina, se compone de 7 ramificaciones (brazos Campanario, Tristeza, Blest, Machete, Rincón, Última Esperanza y Huemul) y una cuenca distal que alberga en sus márgenes a las ciudades de San Carlos de Bariloche y Dina Huapi (Fig. 1). La región se halla bajo la influencia del sistema de subducción localizado en el borde oriental del Oceáno Pacífico Sur (placas de Nazca y Sudamericana), por lo cual está expuesta a una actividad volcánica frecuente caracterizada por erupciones explosivas de los volcanes andinos, que provocan recurrentes caídas de ceniza volcánica transportada por los vientos dominantes del oeste. Paralelamente, según información proporcionada por el Instituto Nacional de Prevención Sísmica de la República Argentina, el sector andino de Patagonia Norte presenta una sismicidad moderada (INPRES, 1991).

El 22 de mayo de 1960, el antiguo muelle del puerto de San Carlos de Bariloche fue destruído durante un evento de tsunami que golpeó las costas de esta localidad. Este evento fue concomitante al terremoto de Valdivia (Chile), el mayor sismo registrado históricamente (9.5 de magnitud). Los mecanismos que desencadenaron esta ola en el lago Nahuel Huapi fueron descriptos por Villarosa et al. (2009), hallándose estrechamente relacionados con 1) la actividad sísmica y volcánica periódica que afecta a la Norpatagonia Andina y 2) las características topográficas de las cubetas glaciares que conforman las cuencas lacustres de la región, cuyas pronunciadas pendientes favorecen la movilización de los sedimentos -glacigénicos, fluviales y piroclásticos- hacia el interior de las cuencas. Los movimientos sísmicos registrados el 22 de mayo de 1960, con epicentro próximo a la ciudad de Valdivia, provocaron en el lago Nahuel Huapi el desplazamiento de importantes volúmenes de sedimentos del lecho en las cercanías de Puerto San Carlos (Fig. 1). Este deslizamiento masivo causó a su vez el desplazamiento de una masa de agua, con la consiguiente generación de una onda tipo tsunami que golpeó las costas de la ciudad, cobrándose 2 vidas humanas y provocando daños en algunas embarcaciones. Flujos de detritos generados en los sedimentos donde se asentaba el antiguo muelle fueron, probablemente, los responsables del colapso de éste (Villarosa et al., 2009). 


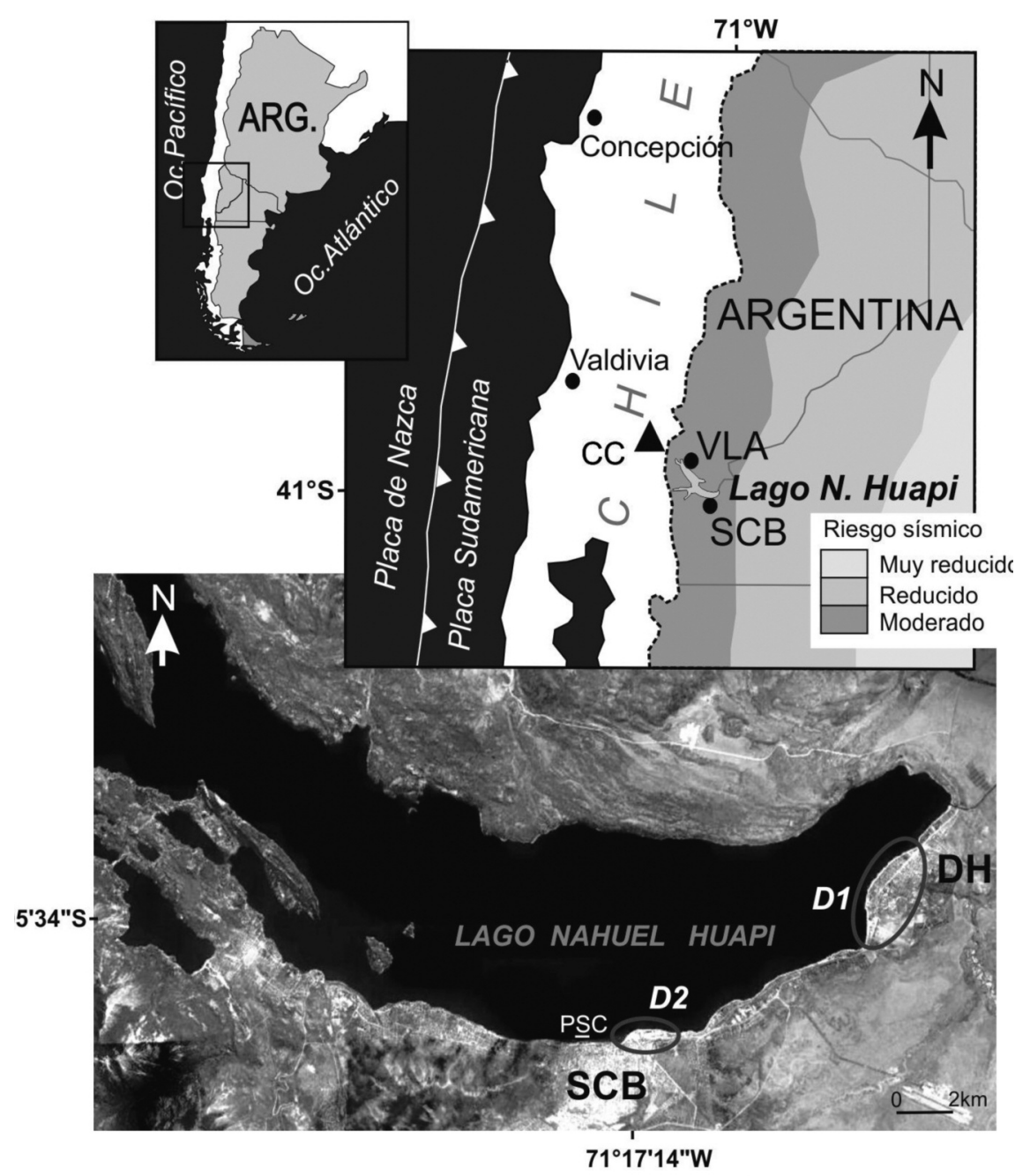

Figura 1. Mapa de ubicación. D1=delta del río Nirihuau; D2=delta del arroyo Nireco. $C C=$ Cordón Caulle; $S C B=S . C$. de Bariloche; VLA=V. La Angostura; DH=Dina Huapi; $P S C=$ Puerto San Carlos. En tonos de gris se indican las áreas de riesgo sísmico en Patagonia Norte argentina.

En los últimos años, en varias ocasiones siempre coincidentes con el registro de sismos en la región, han habido reportes de oscilaciones de baja frecuencia en el nivel del lago Nahuel Huapi: en mayo de 2008, simultáneamente al registro de actividad sísmica en el complejo volcánico Puyehue-Cordón Caulle; el 27 de febrero de 2010, coincidente con el terremoto de magnitud 8.8 con epicentro próximo a Concepción (Chile); y el 11 de julio de 2011, mientras se registraba actividad sísmica y volcánica en el Cordón 
Caulle. Se han establecido 2 hipótesis respecto a la naturaleza de este fenómeno: 1) se trata de olas tipo seiche producto de los movimientos sísmicos, o 2) al igual que lo ocurrido en 1960, son ondas generadas por deslizamientos subacuáticos (dado que no existen evidencias de movimientos en masa subaéreos) que recurrentemente provocan desplazamientos en la masa de agua. Con el objeto de verificar la segunda hipótesis, se está investigando la posible ocurrencia de fenómenos de remoción en masa en el lecho lacustre, con especial énfasis en las áreas cercanas a las localidades costeras.

La localidad de Bariloche y su vecina Dina Huapi han experimentado un rápido crecimiento poblacional a lo largo de las últimas décadas. Dicho crecimiento, sumado a la fuerte actividad turística que desarrollan ambas localidades, generó una ocupación cada vez más intensa de los espacios costeros del lago Nahuel Huapi, incorporando al espacio urbano ambientes particularmente activos, entre los que se cuentan los deltas lacustres del río Ñirihuau (D1) y del arroyo Ñireco (D2) (Fig. 1). Ello ha incrementado la vulnerabilidad de las áreas costeras del lago (entendida como el grado de pérdida de los elementos expuestos, según definición de Varnes, 1984) frente a la ocurrencia de deslizamientos subacuáticos y tsunamis. El presente trabajo tiene como objetivo identificar y caracterizar los fenómenos de remoción en masa en la porción sumergida de estos deltas lacustres, teniendo en cuenta que una ola generada a partir de un amplio deslizamiento en estos ambientes deltaicos podría afectar a costas vulnerables de estas localidades.

\section{Metodología}

Mediante un Sonar Batimétrico por Medición de Fase (GeoSwath Bathymetry System Plus, GeoAcoustics Ltd.) con transductores de $250 \mathrm{kHz}$ montado en una embarcación guardacostas de la Prefectura Naval Argentina, se estudió la morfología de la porción sumergida de los deltas del río Ñirihuau y del arroyo Ñireco. Este equipo permite registrar la batimetría del lago con alta resolución, por fajas, hasta una profundidad de unos 120 m, límite de operación del sistema. La georreferenciación de los datos batimétricos se efectuó mediante GPS diferencial, para lo cual se estableció una estación terrestre de referencia en Puerto San Carlos (Fig. 1). El estudio se efectuó mediante transectas aproximadamente paralelas a la línea de costa, con una superposición lateral del 50\% entre fajas de barrido. De esta manera se cubrió un área de $\sim 5.1 \mathrm{~km}^{2}$ en D1 y $\sim 1.7 \mathrm{~km}^{2}$ en D2. Los datos fueron procesados con el software GeoSwath Plus para producir una matriz de datos $\mathrm{x}, \mathrm{y}, \mathrm{z}$ corregida y georreferenciada, con una resolución de 3 x $3 \mathrm{~m}$. A partir de éstas se confeccionaron los Modelos Digitales de Elevación en Surferv8 y los mapas derivados de pendientes, curvas de nivel, orientación de laderas y sombreado del relieve, mediante el software ArcGIS v8.

\section{Resultados}

Perfiles batimétricos trazados en dirección aproximadamente perpendicular a la línea de costa (Fig. 2) muestran tres ambientes diferenciados en los deltas estudiados. Bordeando la planicie deltaica subaérea se extiende una plataforma somera ( $\leq 10 \mathrm{~m}$ de profundidad) de pendientes levemente inclinadas hacia el interior de la cuenca lacustre, cuyo ancho puede 
llegar a superar los $150 \mathrm{~m}$ en algunos sectores (Fig. 2, perfil b-b'). Las mayores pendientes de la plataforma costera se observan en D2, con valores que oscilan entre los $0.4^{\circ}$ y $6^{\circ}$. Luego la topografía se hace más abrupta, marcando el inicio del frente deltaico. El punto de inflexión entre estos dos ambientes se ubica entre los 4 y $8 \mathrm{~m}$ de profundidad en D1 y entre los 6 y 10 m de profundidad en D2. Los frentes de delta, con un ancho de unos 190-280 m en D1 y de $100-270 \mathrm{~m}$ en D2, presentan fuertes pendientes $\left(13.9^{\circ} \pm 1.6^{\circ}\right.$ y $17.4^{\circ} \pm 1.7^{\circ}$ en D1 y D2, respectivamente). Entre los 50 y los $100 \mathrm{~m}$ de profundidad, se observa en todos los casos un cambio de pendiente que se interpreta como el inicio del prodelta, donde las pendientes se reducen a $5.5^{\circ} \pm 0.6^{\circ}$ y $9^{\circ} \pm 1.2^{\circ}$ en D1 y D2, respectivamente.
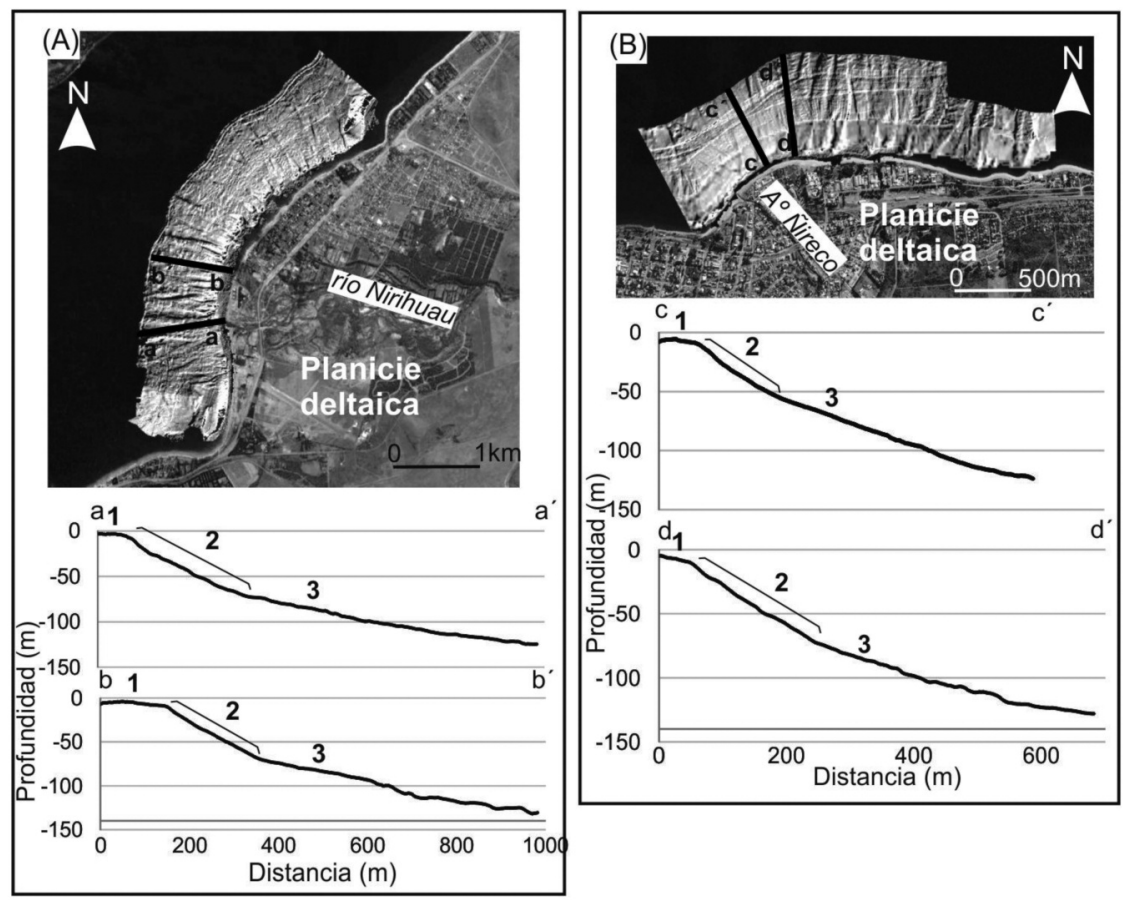

Figura 2. Ambientes identificados en los deltas del río Nirihuau (A) y del arroyo Nireco (B). $1=$ plataforma somera (¿terraza lacustre?); $2=$ frente de delta; $3=$ prodelta. Exageración vertical=2x.

En ambos deltas se identificaron numerosas áreas movilizadas, producto de deslizamientos ocurridos en el sector frontal, que provocan un retroceso progresivo de la plataforma costera (Figs. 3 y 4). La cercanía entre estos eventos de remoción en masa -cuyas cabeceras en algunos casos llegan a coalescer entre sí (Fig. 3)- generalmente impide una clara delimitación del área movilizada por cada evento pendiente abajo de los sectores de arranque. No obstante, la existencia de extensas escarpas laterales de unos 800-900 m (D1) y 600-700 m (D2) de longitud permite identificar algunos de estos eventos hasta el límite del área estudiada, donde el mayor de éstos afectó una superficie de $\sim 0.13 \mathrm{~km}^{2}$ (Fig. 5). 

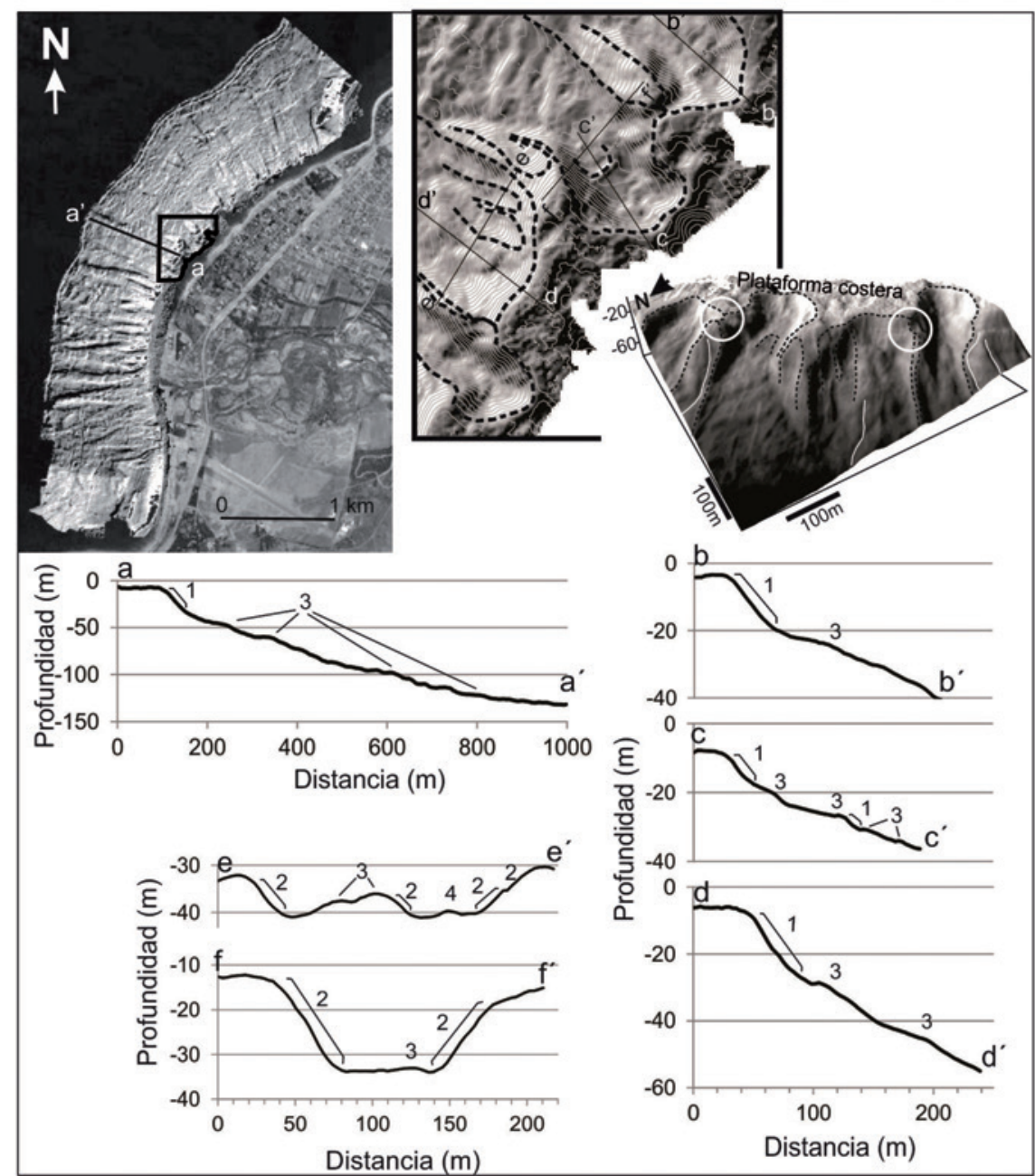

Figura 3. Deslizamientos subacuáticos en el delta del río Nirihuau que provocan un retroceso avanzado de la plataforma costera. En línea punteada se indican las escarpas de deslizamientos y las líneas blancas representan los canales subacuáticos. Los círculos blancos muestran la coalescencia entre cabeceras contigüas. $1=$ escarpas de cabecera; $2=$ escarpas laterales; $3=$ material desplazado;

$4=$ sedimentos no movilizados. Exageración vertical $=2 x$ (perfil a) y $3 x$ (perfiles $b, c, d, e, f)$.

Para caracterizar los sectores de arranque se seleccionaron las áreas más movilizadas en D1 y D2 (Figs. 3, 4 y 5). En estos sectores, las escarpas de cabecera muestran una altura promedio superior a los $10 \mathrm{~m}$ y una longitud que puede alcanzar los $200 \mathrm{~m}$ en ambos deltas. Presentan superficies cóncavas y pendientes que superan los $20^{\circ}$, alcanzando valores de hasta $28^{\circ}$ en algunos sectores (Fig. 3, perfil d-d'). Las escarpas laterales, con alturas del orden de 4 a 20 m (D1) y de 2 a 6 m (D2), presentan pendientes que oscilan entre los $9^{\circ}$ y $24^{\circ}$ en D1 y los $15^{\circ}$ y $24^{\circ}$ en D2. La corona semicircular, el perfil 
cóncavo de las escarpas de cabecera y la existencia de depósitos próximos a éstas sugieren deslizamientos rotacionales. Sin embargo, la existencia de algunas extensas escarpas laterales identificadas hasta el límite del área estudiada indicaría que estos deslizamientos, generados en principio como movimientos rotacionales, habrían evolucionado posteriormente a deslizamientos traslacionales que movilizaron parte del material a través del prodelta hacia áreas más profundas de la cuenca lacustre. En el interior de algunos deslizamientos se desarrollan movimientos en masa más localizados (Figs. 3 y 4). Probablemente se trata de deslizamientos rotacionales dada la geometría del plano de despegue y la cercanía de los depósitos a los sectores de arranque (Fig. 3, perfil c-c’').

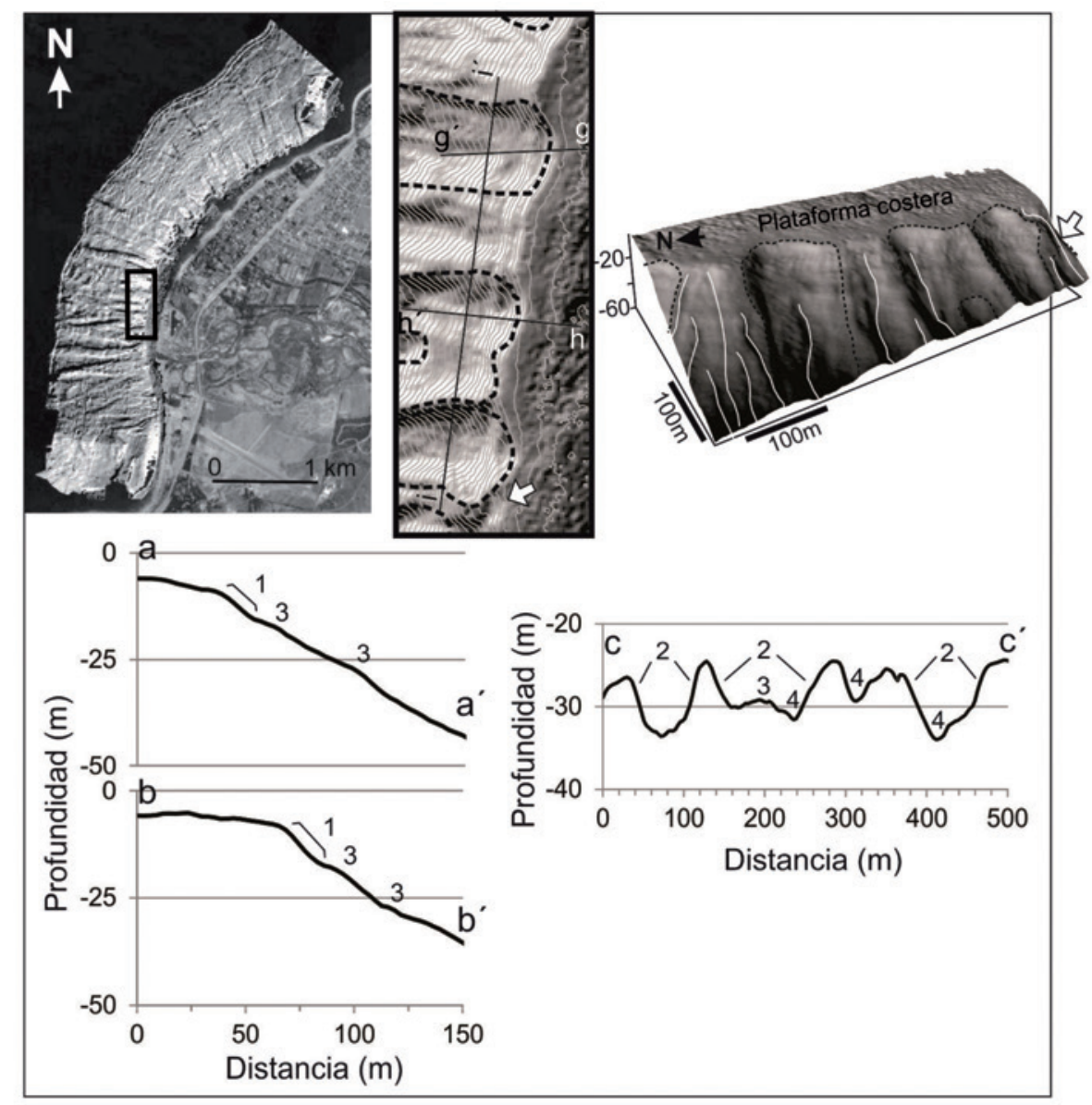

Figura 4. Deslizamientos subacuáticos en el delta del río Nirihuau. Se muestra un sector con retroceso incipiente de la plataforma costera. En línea punteada se indican las escarpas de deslizamientos y las líneas blancas representan los canales subacuáticos. La flecha blanca señala el canal que retrocede de una escarpa de deslizamiento. $1=$ escarpas de cabecera; $2=$ escarpas laterales; $3=$ material desplazado; $4=$ canales subacuáticos. Exageración vertical $=2 x$ (perfiles $a, b)$ y $10 x$ (perfil c). 
Canales subacuáticos generados por procesos gravitacionales (flujos de detritos y/o corrientes de turbidez) también surcan los frentes deltaicos (Figs. 3, 4 y 5), contribuyendo en algunos casos al retroceso de la plataforma costera. Sus longitudes varían entre 40800 m en D1 y 60-400 m en D2. Los más profundos presentan paredes laterales que superan los $5 \mathrm{~m}$ de altura en algunos sectores. Estas geoformas ya han sido identificadas en otros sectores del lecho frente a la ciudad de San Carlos de Bariloche (Villarosa et al., 2009; Beigt et al., 2010) y en deltas lacustres ubicados en las cercanías de la localidad de Villa La Angostura (Fig. 1) (Beigt et al., 2012). En el área de estudio de este trabajo, se han identificado canales tanto en el interior de las áreas movilizadas como fuera de éstas, en ocasiones cortando las escarpas de deslizamientos (Fig. 4). Su origen probablemente está vinculado a una combinación de diversos factores; 1) la licuefacción de parte del material desplazado por los deslizamientos, 2) el retroceso de las escarpas de deslizamiento o 3) el ingreso al lago de flujos hiperconcentrados desde el sistema fluvial.

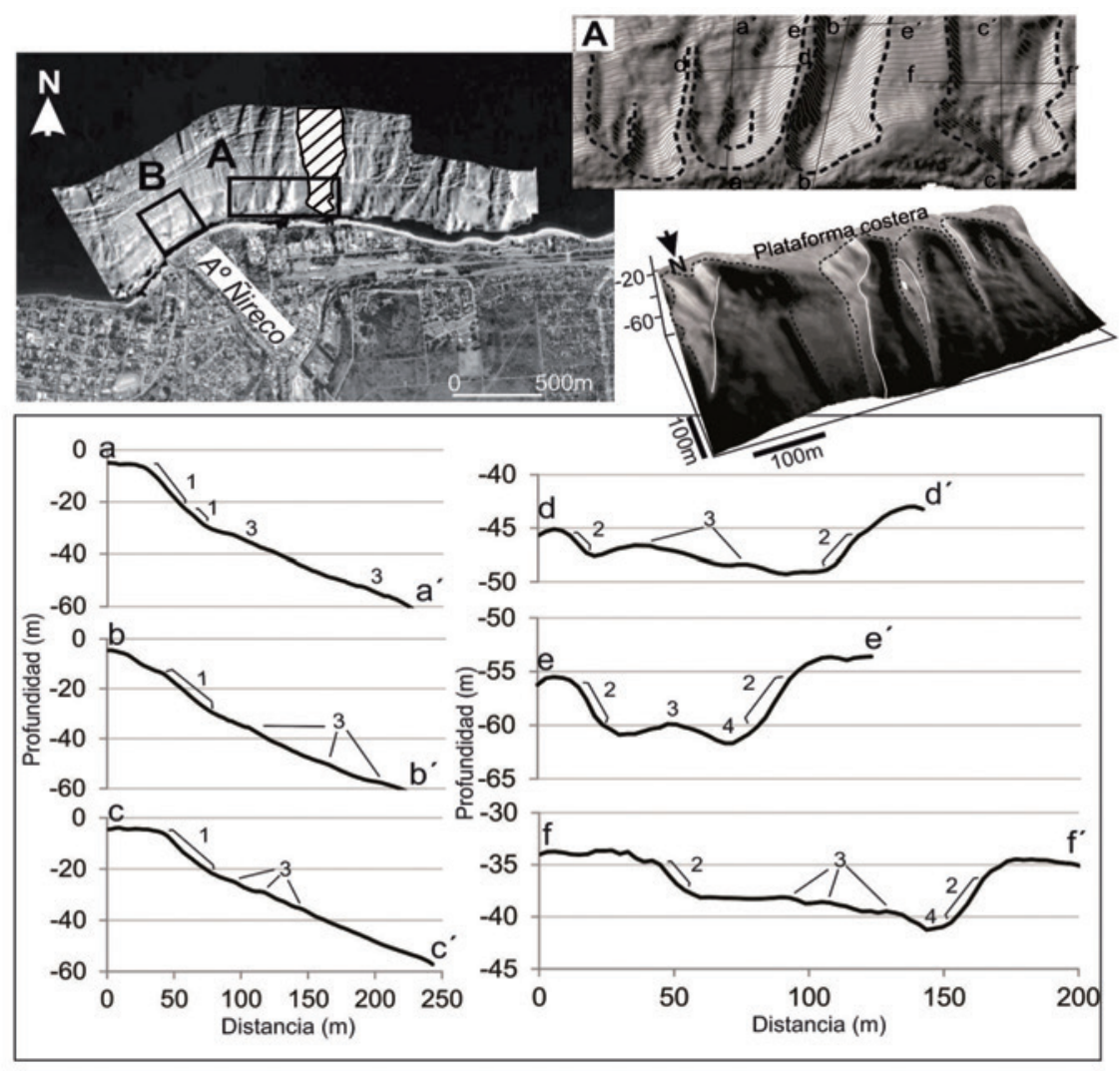

Figura 5. Deslizamientos subacuáticos en el delta del arroyo Nireco. La superficie tramada indica el mayor evento identificado. (A) Sectores de arranque seleccionados. (B) Ubicación de las geoformas deposicionales de la figura 6 . Las líneas punteadas señalan las escarpas de deslizamientos y las líneas blancas representan los canales subacuáticos. $1=$ escarpas de cabecera; 2 = escarpas laterales; $3=$ material desplazado; $4=$ canales subacuáticos . Exageración vertical $=2 x$ (perfiles $a, b, c)$ y $4 x$ (perfiles $d, e, f)$. 
En este sentido, cabe mencionar que frente a la actual desembocadura del arroyo Nireco se hallaron unas geoformas deposicionales que probablemente estén asociadas a la dinámica de éste en su ingreso al cuerpo lacustre (Figs. 5B y 6). Presentan una apariencia de depósitos en forma de abanico, cuyos ápices se ubican entre los 10 y los $36 \mathrm{~m}$ de profundidad aproximadamente. Su altura oscila entre los 4 y los $9 \mathrm{~m}$ (Fig. 6, perfiles a-a' y b-b'). El perfil longitudinal de estos "abanicos" subacuáticos es convexo en su sector proximal (registrando pendientes máximas de $22^{\circ}$ ) y cóncavo en el área distal (Fig. 6, perfil d-d’). Numerosos canales subacuáticos surcan la superficie de estas geoformas, profundizando algunos de sus bordes laterales (Fig. 6, perfiles a-a' y b-b'). La bibliografía señala que en los ambientes lacustres, los sistemas de flujos de fondo generados en zonas relacionadas con desembocaduras permiten el desarrollo de abanicos subacuáticos (Anadón Monzón, 1984). En este trabajo, sin embargo, no se observaron evidencias de canales a través de los cuales se hubiese podido transportar este material hasta estas profundidades. Escarpas de deslizamientos ubicadas en el sector distal de la plataforma costera, pendiente arriba de algunos de estos depósitos, completan un panorama complejo. Investigaciones en curso acerca de las características sedimentológicas de estas geoformas permitirán establecer con mayor precisión el mecanismo específico que las genera.

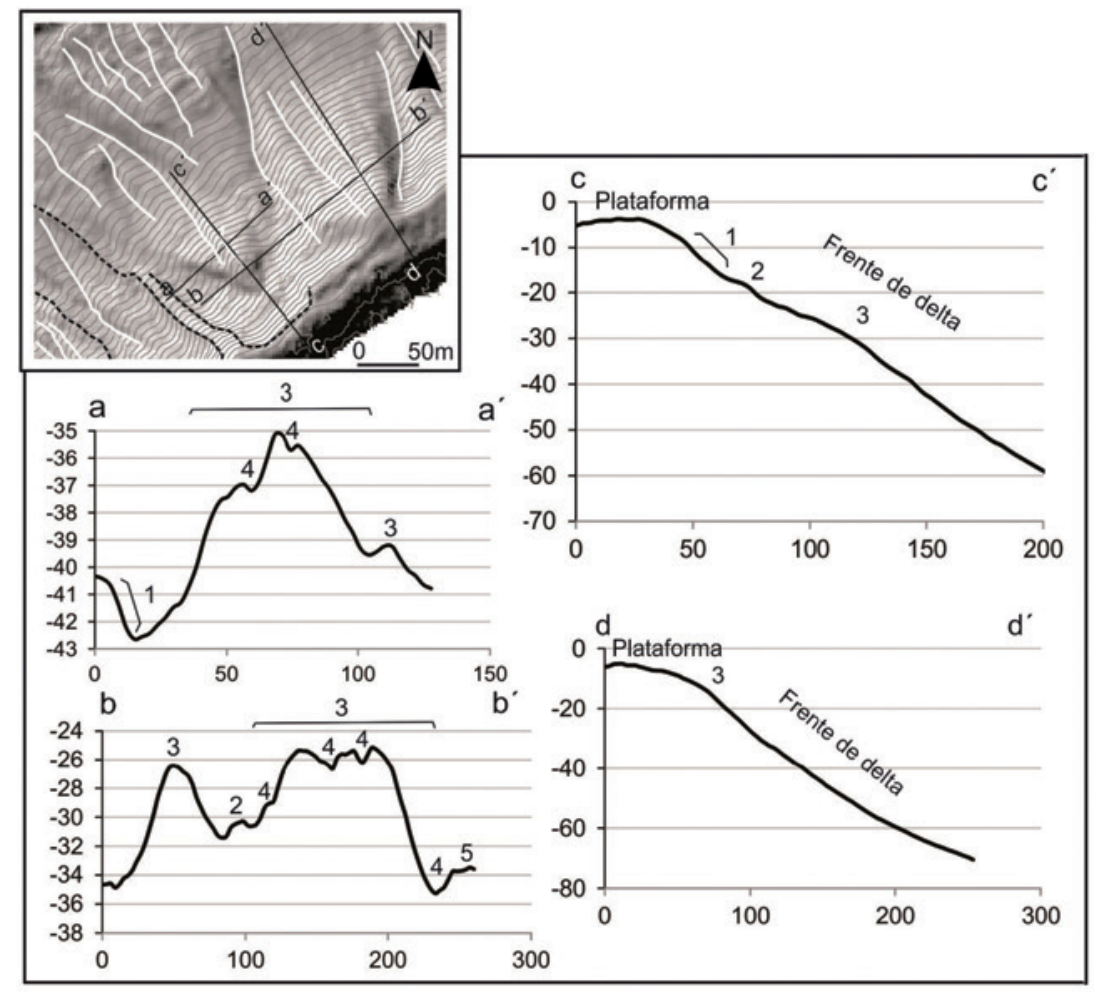

Fig. 6. Geoformas deposicionales frente a la desembocadura del arroyo Nireco (ubicación en Figura $5 B$ ). Las líneas punteadas indican escarpas de deslizamientos. $1=$ escarpas de deslizamientos; $2=$ depósitos de remoción en masa?; 3 = abanicos? subacuáticos; 4= canales subacuáticos; 5= área no movilizada. Exageración vertical $=10 x$ (perfiles $a, b)$ y $2 x$ (perfiles $c, d$ ). 


\section{Discusión}

Los ambientes estudiados en el presente trabajo muestran rasgos característicos de deltas tipo Gilbert. Estos deltas se caracterizan por presentar tres ambientes deposicionales: una planicie deltaica subaérea donde dominan los procesos aluviales, un frente deltaico de pendientes pronunciadas donde el sustrato se moviliza mediante procesos gravitacionales y un prodelta suavemente inclinado donde se deposita el material más fino, transportado en suspensión (Postma y Roep, 1985; Nemec, 1990). En los deltas estudiados pudieron identificarse los frentes deltaicos, hallándose efectivamente abundancia de procesos gravitacionales y pendientes abruptas que se corresponden con el rango citado en la bibliografía para los frentes de delta de Gilbert $\left(10^{\circ}-25^{\circ}\right.$, dependiendo de las características del sedimento, Postma y Roep, 1985). Bordeando el frente de delta en su sector distal, se identificó el ambiente de prodelta, donde se observan depósitos de deslizamientos iniciados en los frentes (Fig. 3, perfil a-a'). Sin embargo, como se mencionó anteriormente, se considera que una parte de estos materiales continuó deslizándose hacia sectores más profundos del lecho, lo cual fue probablemente favorecido por las pendientes que presentan los prodeltas (Fig. 2). En este sentido, trabajos previos en el lago Nahuel Huapi (Chapron et al., 2006; Villarosa et al., 2009) mostraron evidencias de una megaturbidita en el sector central de la cuenca lacustre, frente a la ciudad de Bariloche (a unos $200 \mathrm{~m}$ de profundidad). Su génesis se vinculó con el gran movimiento en masa subacuático que provocó el tsunami de 1960. Este depósito dista unos 3000 m aproximadamente de las escarpas de deslizamientos que le dieron origen, lo cual indica que los materiales movilizados por procesos de remoción en masa iniciados en los ambientes costeros pueden recorrer grandes distancias, transportados por corrientes de turbidez que descienden las elevadas pendientes hasta depositar los sedimentos en los sectores más profundos y planos de la cubeta lacustre.

La extensión de las escarpas de cabecera - de unos 10 m de altura y hasta 200 m de longitud- y de las escarpas laterales (algunas de las cuales se han identificado hasta el límite del área estudiada, con longitudes que superan los $600 \mathrm{~m}$ ), sumada a la abundancia de deslizamientos, indican que en los deltas lacustres estudiados se desarrollan periódicamente eventos de remoción que involucran grandes volúmenes de sedimentos. Estos eventos, que movilizan una importante cantidad de materiales a través de grandes distancias, son potenciales generadores de ondas en la superficie del lago.

Entre la llanura deltaica subaérea y el frente de delta se extiende una amplia plataforma (¿terraza lacustre?), presente en ambos deltas estudiados. La formación de terrazas debido a fluctuaciones en el nivel de un lago o mar es un fenómeno geomorfológico ampliamente observado (Büdel, 1982; Yefimova y Mats, 2003, entre otros). Las olas de tormenta producen superficies erosivas, siendo la erosión particularmente intensa durante los períodos de incrementos significativos del nivel de las aguas, debido al desplazamiento de la zona de rompiente hacia sectores más elevados, previamente emergidos (Dabrio, 2000). El nivel del lago Nahuel Huapi muestra grandes variaciones, tanto estacionales como interanuales (Pereyra, 2007). Es probable que la formación de las terrazas lacustres en los deltas estudiados se halle vinculada a la abrasión de las plani- 
cies deltaicas por olas de tormenta durante los períodos de niveles altos del lago. Plataformas similares a las presentadas en este trabajo han sido identificadas previamente en deltas lacustres cercanos a la localidad de Villa La Angostura (Fig. 1), donde además, un segundo nivel de terraza de unos $5 \mathrm{~m}$ de ancho hallado en el sector distal de una de estas plataformas sugeriría un inicio de abrasión de este ambiente (Beigt et al., 2012). La presencia de esta plataforma de abrasión en ambos deltas estudiados sugiere que se trata de deltas tipo Gilbert retrabajados por olas.

\section{Conclusiones}

Este estudio se enfocó en la identificación y caracterización de los fenómenos de remoción en masa desarrollados en los frentes de deltas lacustres del lago Nahuel Huapi con el objeto de 1) aportar información relevante que sirva de base a futuras evaluaciones de peligrosidad en las costas de San Carlos de Bariloche y Dina Huapi, 2) acercar una posible explicación de las oscilaciones de nivel que recurrentemente se registran en la superficie del lago de manera simultánea a terremotos producidos a nivel regional. Los resultados de este trabajo indican que en los ambientes deltaicos del lago Nahuel Huapi se desarrollan periódicamente eventos de remoción que involucran grandes volúmenes de sedimentos. Parte del material desplazado es aparentemente transportado en forma canalizada mediante corrientes de densidad hacia sectores más profundos de la cuenca lacustre. Es altamente probable que estos eventos de magnitud considerable, que movilizan una importante cantidad de materiales a través de grandes distancias, generen ondas en la superficie del lago.

Las olas de tormenta durante los períodos de niveles altos del lago parecen constituir un agente que modifica sustancialmente la morfología subacuática de los deltas del lago Nahuel Huapi, conformando una plataforma de abrasión que se extiende entre la planicie subaérea y el frente deltaico. Paralelamente, geoformas deposicionales halladas frente a la desembocadura del arroyo Nireco sugieren una alta sedimentación a partir de los aportes del sistema fluvial. Mediante análisis sedimentológicos y estudios sísmicos actualmente en curso se espera poder establecer con precisión los mecanismos que han dado origen tanto a las "terrazas" como a los "abanicos" subacuáticos. Con el fin de evaluar la peligrosidad que estos eventos de remoción en masa pueden representar para las localidades de Bariloche y Dina Huapi, se pretende continuar el monitoreo de los frentes deltaicos para observar posibles cambios en su morfología, identificar áreas potencialmente inestables y modelar el comportamiento de la masa de agua durante un deslizamiento de magnitudes importantes.

\section{Agradecimientos}

Este trabajo contó con la financiación de la Agencia Nacional de Promoción Científica y Tecnológica (PICT 2010-0636, PICT 2010-2046) y del Ministerio de Ciencia, Tecnología e Innovación Productiva (PROEVO 40-B-187, PROEVO 40-B-188). Los autores de este trabajo agradecen el apoyo logístico de la Prefectura Naval Argentina (San Carlos de Bariloche) en la realización de las campañas al área de estudio. 


\section{Referencias}

Anadon Monzon, P. 1984. Sedimentación lacustre. En Ciclo de seminarios de sedimentología, Vol.1, V. Gabaldon (coord), Instituto Geológico y Minero de España, Madrid, pp. 93-130.

Baster, I., Girardclos, S., Wildi, W., Pugin, A. 2003. High-resolution seismic stratigraphy of a Holocene lacustrine delta fan in western Lake Geneva (Switzerland). Eclogae Geologicae Helvetiae 96 (supplement 1), S11-S20.

Beigt, D., Villarosa, G., Gómez, E.A. 2012. Deslizamientos subacuáticos en sistemas deltaicos del lago Nahuel Huapi: resultados preliminares de una evaluación de peligrosidad para la localidad de Villa La Angostura (Neuquén). En Actas de las $2^{a s}$ Jornadas Nacionales de Investigación y Docencia en Geografía Argentina y $8^{a s}$ Jornadas de Investigación y Extensión del Centro de Investigaciones Geográficas, Centro de Investigaciones Geográficas y Universidad Nacional del Centro, Tandil, pp. 1-7.

Beigt, D., Villarosa, G., Gómez, E.A. 2010. Mass-wasting events and related morphology along a coastal portion of Nahuel Huapi lake bed (Patagonia). En $18^{\text {th }}$ International Sedimentological Congress, Abstracts Volume, IAS, Mendoza, p. 166.

Büdel, J. 1982. Climatic Geomorphology. Princeton University Press, Princeton, 443 pp.

Chapron, E., Ariztegui, D., Mulsow, S., Villarosa, G., Pino, M., Outes, V., Juvignie, E., Crivelli, E. 2006. Impact of the 1960 major subduction earthquake in Northern Patagonia (Chile, Argentina). Quaternary International 158, 58-71.

Dabrio, C.J. 2000. Coastal sedimentary environments and sea level changes. Ciencias da Terra $14,39-54$.

Girardclos, S., Schmidt, O.T., Sturm, M., Ariztegui, D., Pugin, A., Anselmetti, F.S. 2007. The 1996 AD delta collapse and large turbidite in Lake Brienz. Marine Geology 241, 137-154.

INPRES. 1991. Reglamento INPRES-CIRSOC 103. Parte I. Normas argentinas para construcciones sismorresistentes. $206 \mathrm{pp}$.

Kremer, K., Simpson, G., Girardclos, S. 2012. Giant Lake Geneva tsunami in AD 563. Nature Geoscience 5, 756-757.

Nemec, W. 1990. Aspects of sediment movement on steep delta slopes. En Coarse Grained Deltas: Special Publications of the International Association of Sedimentologists 10, A. Colella, D.B. Prior (eds.), The International Association of Sedimentologists, Blackwell Scientific Publications, pp. 29-73.

Pereyra, F.X. 2007.Geomorfología urbana de San Carlos de Bariloche y su influencia en los peligros naturales. Revista de la Asociación Geológica Argentina 62 (2), 309-320.

Postma, G., Roep, T.B. 1985. Resedimented conglomerates in the bottomsets of Gilbert-type gravel deltas. Journal of Sedimentary Petrology 55 (6), 874-885.

Varnes, D.J. 1984. Landslides hazard zonation: a review of principles and practice. Natural Hazards 3, Commission on landslides of the IAEG, UNESCO, $61 \mathrm{pp}$.

Villarosa, G., Outes, V., Gómez, E.A., Chapron, E., Ariztegui, D. 2009. Origen del tsunami de mayo de 1960 en el lago Nahuel Huapi, Patagonia: aplicación de técnicas batimétricas y sísmicas de alta resolución. Revista de la Asociación Geológica Argentina 65 (3), 593-597.

Yefimova, I.M., Mats, V.D. 2003. Change of the Baikal Lake level substantiated by analysis of terraces. Berliner Paläobiologische Abhandlungen 4, 77-87. 